\title{
Correction to: Effect of Er:YAG laser etching on topography, microstructure, compressive strength, and bond strength of a universal adhesive to calcium silicate cements
}

\author{
Fereshteh Shafiei ${ }^{1}$ (D) $\cdot$ Paria Dehghanian $^{2} \cdot$ Mahtab Memarpour $^{3} \cdot$ Mohammad Sabeti $^{4}$
}

Published online: 16 October 2019

(C) Springer-Verlag GmbH Germany, part of Springer Nature 2019

\section{Correction to: Clinical Oral Investigations}

https://doi.org/10.1007/s00784-019-02903-2

Figure 2 was incorrect in the original published version of this article. Correct figure in presented here.

The original article has been corrected.

Publisher's note Springer Nature remains neutral with regard to jurisdictional claims in published maps and institutional affiliations.

The online version of the original article can be found at https://oi.org/ 10.1007/s00784-019-02903-2

\section{Mahtab Memarpour}

memarpour@sums.ac.ir

1 Oral and Dental Disease Research Center, Department of Operative Dentistry, School of Dentistry, Shiraz University of Medical Sciences, Shiraz, Iran

2 Student Research Committee, School of Dentistry, Shiraz University of Medical Sciences, Shiraz, Iran

3 Oral and Dental Disease Research Center, Department of Pediatric Dentistry, School of Dentistry, Shiraz University of Medical Sciences, Shiraz, Iran

4 Advanced Specialty Education Program in Endodontics Diploma, American Board of Endodontics, Department of Endodontics, School of Dentistry, University of California, San Francisco, CA, USA 
Fig. 2 SEM images of Biodentine (a-e) along with AFM images in two views, 3D (a1-e1) and topography (a2-e2). a Non-etch surfaces, gelform structure with globular particles, and some microchannels ( $\mathrm{mc})$; a 1 , a 2 microretentive view with a number of concavities and convexities. b Acid-etch surface, eroded surface as squaredshaped (sq) view; b1, b2 surface with microroughness similar to non-etch surface. c Laser-etch 60, gel-form matrix (gm) with crystalline structure (crs), microchannels $(\mathrm{mc})$, reformed globular particles (rgp), and selective loss of matrix in some area; $\mathrm{c} 1, \mathrm{c} 2$ microretentive pattern similar to aid-etch surface. d Laseretch 80 surface, selective loss of surface exposing gelform matrix (gm), an area of crystalline structure (crs) and needle-like crystals (ncrs) with few microchannels $(\mathrm{mc})$, and evident crack (crk); d1, d2 surface irregularities similar to non-etch surface, except of presence of crack. e Laser-etch 100, more loss of some surface and cracked surface with few crystals and needle-like crystal (ncrs), and few microchannels; e1, e2 surface roughness similar to laser-etch 80 and deep crack

\section{a1}

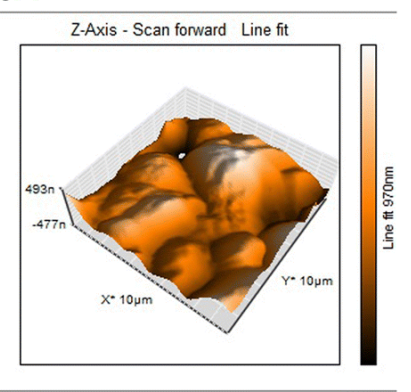

b1

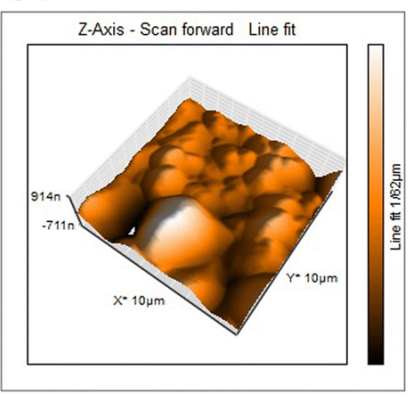

c1

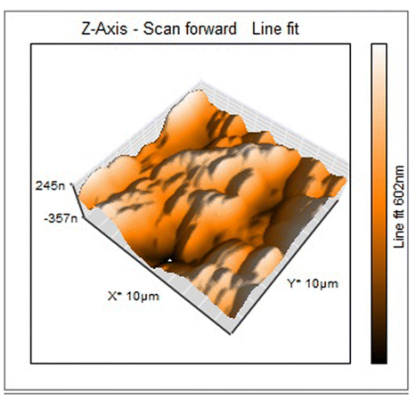

d1

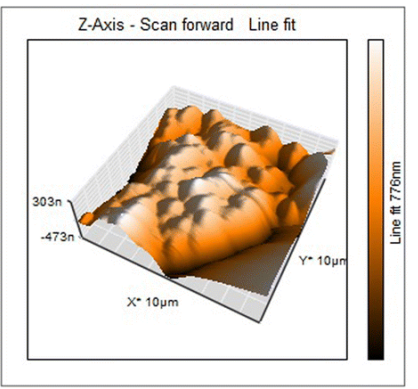

e1

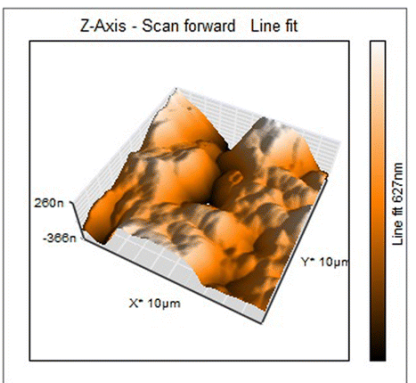

a2

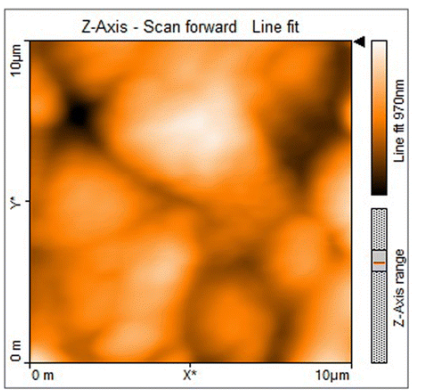

b2

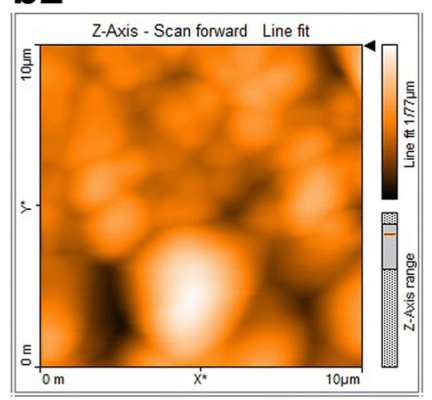

c2

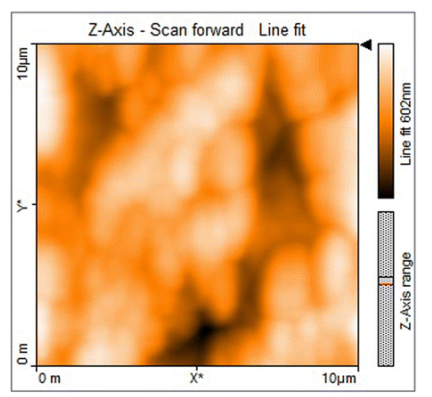

d2

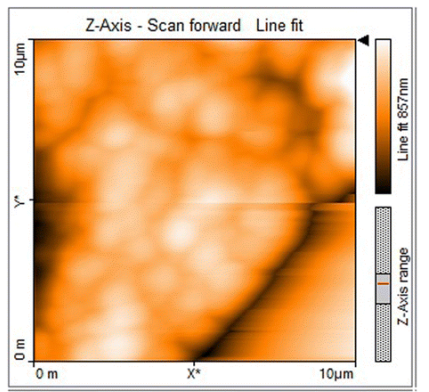

e2

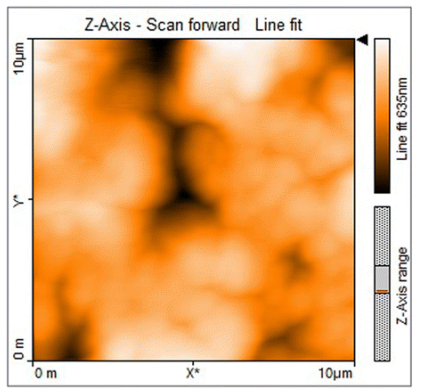

